\title{
RESPONSES OF ROOTSTOCKS TO NUTRIENT INDUCED HIGH EC LEVELS ON YIELD AND FRUIT QUALITY OF GRAFTED TOMATO CULTIVARS IN GREENHOUSE CONDITIONS
}

\author{
SOYLEMEZ, S. ${ }^{*}$ - PAKYUREK, A. Y. \\ Harran University, Faculty of Agriculture, Department of Horticulture, Sanlurfa, Turkey \\ (phone: +90-505 2413994 ) \\ *Corresponding author \\ e-mail: ssoylemez@harran.edu.tr \\ (Received $16^{\text {th }}$ Dec 2016; accepted $16^{\text {th }}$ Mar 2017)
}

\begin{abstract}
This study aimed to determine the effects of different electrical conductivity (EC) levels and different rootstocks on efficiency and yield and some fruit quality parameters. The study was conducted in a closed supply system with soilless culture. EC levels were prepared by adding all macro and micro plant nutrients to the irrigation water. In the trial, 5 different nutrient- levels with 2

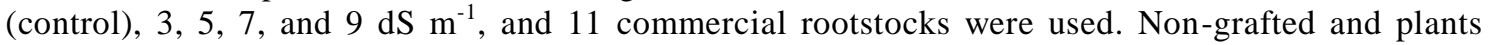
which were grafted upon themselves were also included in the study. The nutrient induced EC levels from $2 \mathrm{dS} \mathrm{m}^{-1}$ to $9 \mathrm{dS} \mathrm{m} ~^{-1}$, total yield decreased by approximately $60 \%$, marketable yield by $63 \%$, total number of fruits by $15 \%$, fruit diameter by $19 \%$, average fruit weight by $45 \%$ and pericarp thickness by $28 \%$ while skin firmness increased $19 \%$, soluble solids amount $45 \%$, lycopene $53 \%$, titratable acidity $96 \%$ and sugars $32 \%$. The effect of rootstocks on yield, fruit size, pericarp thickness and skin firmness, the amount of total soluble solids (TSS), lycopene and reducing sugars were not significant. High EC level had a negative effect on yield and some yield-related fruit properties, whereas it had a positive effect on the amount of TSS, lycopene, titratable acidity and reducing sugar content in addition to improving overall quality. Different EC levels had effects on the yield and quality of tomato, whereas rootstocks had no effect.
\end{abstract}

Keywords: electrical conductivity, salinity, Solanum lycopersicon, soilless culture, abiotic stress

\section{Introduction}

Tomato, the second most important product after potato in the world, is an excellent source of health promoting agents due to the balanced mixture of flavonoids, lutein, B-carotene, lycopene, vitamins $\mathrm{C}$ and $\mathrm{E}$, antioxidants and minerals in its content (Dorais et al., 2008). The chemical contents of tomatoes grown in the greenhouse out of season are negatively affected by factors such as low temperature, low light intensity and duration, feeding disorders and hormone use. Krauss et al. (2007) reported that the desired properties in fresh tomatoes improved when exposed to salt, offering the explanation that salt stress could serve as an alternative method for improving quality. Researchers have reported that high fruit quality is increasingly important for consumers and the market. Higher fruit quality may economically compensate the inevitable yield and fruit weight reduction which occur as a result of salinity.

Cuartero and Fernandez-Munoz (1999) classified the resistance of tomato to salinity as moderately sensitive. Salinity negatively affects almost every aspect of the biochemistry and physiology of the plant and reduces the yield significantly (Cuartero et al., 2006). The ability of crops to grow on saline soils varies among species and depends on the concentration of salts present in the root zone and on 
various environmental and cultural conditions (Mass, 1993). Salt added to nutrient solution is an easy method that can improve tomato fruit quality, but plant growth and fruit production are negatively affected (Zhang et al., 2016). Growers in recent years have attempted to develop water management practices that maintain yields but impose a moderate, controlled level of stress on their crops with the aim of improving fruit quality (Mitchell et al., 1991). Salinity induced by fertilizers, just as the salinity induced by other toxic ions $(\mathrm{Na}, \mathrm{Cl}$, etc.), has ionic and osmotic effects and negatively affects plant growth (Eraslan et al., 2008).

The possibility of applying grafting to improve fruit quality has been scarcely investigated (Flores et al., 2010). Grafting has important effects on the performance of the scion under various growing conditions (Aloni et al., 2010). Grafting is considered to be an environmentally friendly technique for reducing the yield loses caused by salinity and/or drought (Wahb-Allah, 2014). The rootstock/scion combination should be selected carefully for optimum fruit quality (Davis et al., 2008). Rootstocks increase tolerance against pathogens, poor water quality, excessively wet soil, drought and microelement toxicity in the soil. Grafting also improves tolerance against low temperature, water and nutrient intake and reduces the incidence of physiological disorders related to fruit quality (Dorais et al., 2008). The responses of grafted vegetables to rootstocks, heavy metals around the roots and stress conditions induced by nutrient status may be different from those of nongrafted plants (Savvas et al., 2010). Grafting susceptible vegetables onto resistant rootstocks is an effective strategy for taking control of the soil-borne diseases and reducing environmental stresses, thus improving yield (Rouphael et al., 2010).

This study, conducted in a closed supply system of soilless culture, was carried out to determine the effects of nutrient induced EC Levels and rootstocks on plant growth, yield and fruit quality properties in grafted tomatoes (Solanum lycopersicum). We think that this research is one of the most comprehensive studies conducted on rootstocks to date.

\section{Materials and Method}

\section{Trial site and plant material}

The trial was conducted during autumn of 2009 and 2010 at Harran University, Faculty of Agriculture (Turkey), in a polycarbon covered greenhouse applying a closed feeding system. Newton $F_{1}$ (Syngenta) tomato type was used as the scion. This type was grafted onto Unifort, Beaufort, Maxifort (De Reuiter), Kemerit, Seven RZ, Kingkong (RejkZwaan), Body (Seminis), Toro (May), Spirit (Nunhems), Heman (Syngenta) and Resistar (Hazera) tomato rootstocks and was also self-grafted onto Newton $F_{1}$.Non-grafted plants were used as control samples. Some properties of the rootstocks are given in Table 1. Plants were grafted according to the tube graft method and planted in $135 \times 25 \mathrm{~cm}$ or further inter-row distances in pots filled with perlite on 25 March 2009 and 6 March 2010. The greenhouse temperature and relative humidity values are given in Fig. 1 . 


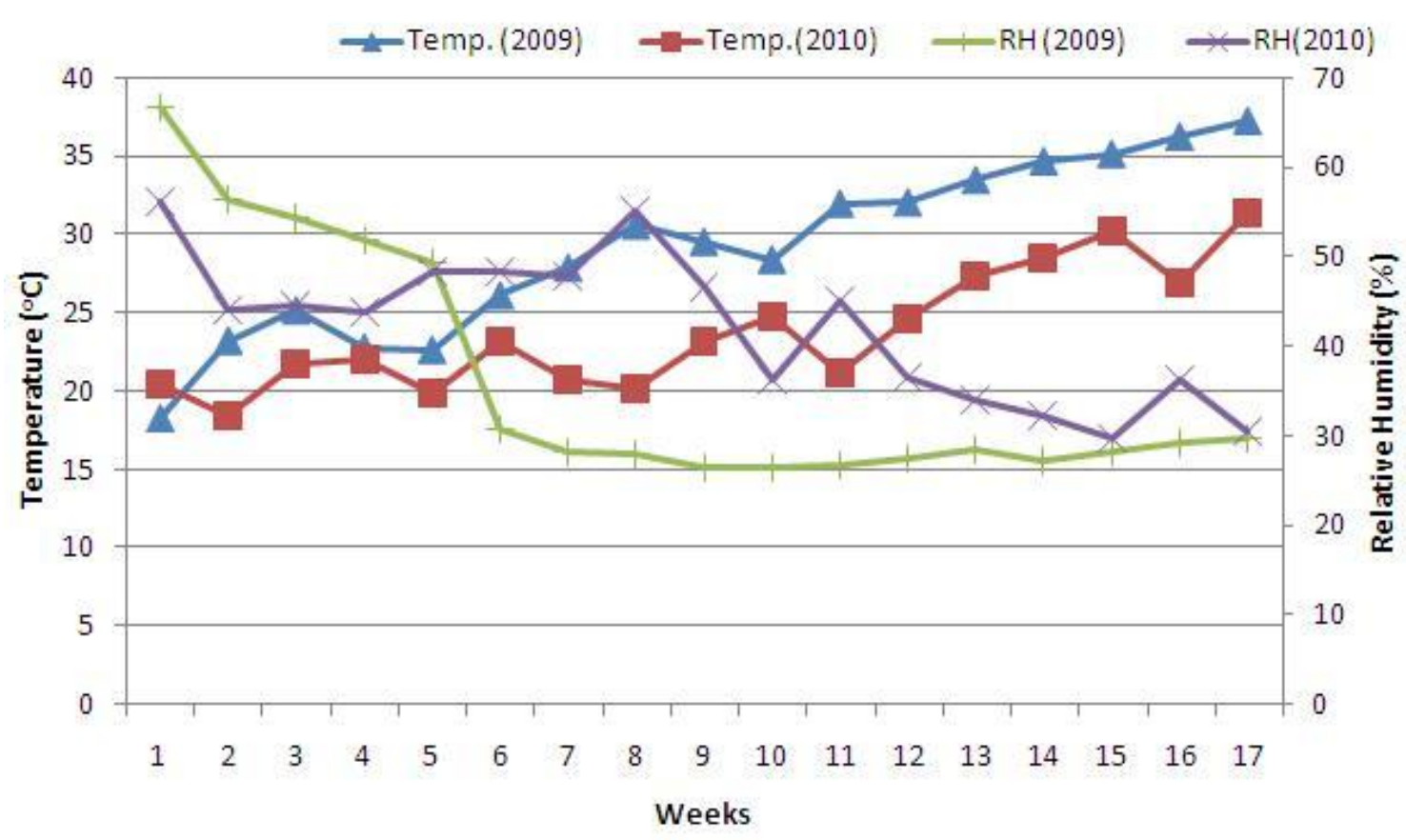

Figure 1. Weekly mean temperature and relative humidity values

Table 1. Properties of rootstocks and scion used in the experiment (Information was provided by seed companies in catalogs and at websites)

\begin{tabular}{|c|c|c|c|}
\hline Name & Product firm & Disease resistant* & \\
\hline Beaufort & De Reuiter & ToMV/Fol:0,1/For/PI/Va/Vd/Ma/Mi/Mj & L. esculentum $\times$ L. hirsutum \\
\hline Maxifort & De Reuiter & ToMV/Fol:0,1/For/PI/Va/Vd/Ma/Mi/Mj & L. esculentum $\mathrm{x}$ L. hirsutum \\
\hline Unifort & De Reuiter & ToMV:0,2/Fol:0,1/For/Va:0/Vd:0/Ma/Mi/Mj & L. esculentum $\mathrm{x}$ L. hirsutum \\
\hline Pegasus & De Reuiter & ToMV/Fol:0.1/Va/Vd/Ma/Mi/Mj & L. esculentum \\
\hline Kemerit & Rejk Zwaan & ToMV/FoI:0,1/PI/Va/Mi & L. esculentum $\mathrm{x}$ L. hirsutum \\
\hline Yedi RZ & Rejk Zwaan & ToMV/FoI:0,1/For/PI/Va/Mi & L. esculentum $\mathrm{x}$ L. hirsutum \\
\hline Kingkong & Rejk Zwaan & ToMV/FoI:0,1/For/PI/Va/Mi & L. esculentum $\mathrm{x}$ L. hirsutum \\
\hline Body & Seminis & ToMV/Fol:2/Pl/Va/Mi & L. esculentum $\mathrm{x}$ L. hirsutum \\
\hline Toro & May & ToMV ,1,2/Fol:1,2/For/Pl/Va/Vd/Ma/Mi/Mj & L. esculentum $\mathrm{x}$ L. hirsutum \\
\hline Spirit & Nunhems & ToMV/Fol:0,1/For/PI/Va/Vd/Ma/Mi/Mj/Pst & L. esculentum \\
\hline Heman & Syngenta & ToMV/Fol:2/For/Va/Mi & L. esculentum \\
\hline Resistar & Hazera & ToMV/Fol:2/For/Va/Mi & L. esculentum \\
\hline
\end{tabular}

*Fol: Fusarium oxysporium (0, 1, 2 races), For: Fusarium oxysporium f.sp. Radicis, V: Verticilium wilt Va: Verticilium albo-atum, Vd: Verticillium dahliae, ToMV: Tomato Mosaic Virus, Pl: root rot, Mi: root-knot nematode (Meloidogyne incognita), Mj: Meloidogyne javanica, Ma: Meloidogyne arenaria, Pst:Pseudomonas syringae pv. Tomato

\section{Nutrient induced EC levels}

In the trial, $5 \mathrm{EC}$ levels, 2 (control), 3, 5, 7, and $9 \mathrm{dS} \mathrm{m}^{-1}$, were used. EC levels were prepared by increasing all nutrient contents (macro and micro). The preparation was given to the plants with a drip irrigation system. The trial was established according to the closed feeding system and reused after EC (Adwa ECO 401 EC meter) and pH (Adwa ECO 200 $\mathrm{pH}$ meter) adjustments to the drained nutrient solution. The old solution was emptied when the EC value of the drained exceeded 1.5-fold of the nutrient solution administered at the beginning and a new nutrient solution was prepared. 


\section{Measurements of yield and fruit}

Diameter and pericarp thickness of the fruit was measured with a digital caliper. All harvested fruits were divided into 5 groups according to their diameter $(>67,57-66,47$ $56,40-46$ and $<40 \mathrm{~mm}$ ), counted and weighed. Fruits with a diameter smaller than 40 $\mathrm{mm}$ and without cracks or blossom end rot were regarded as marketable. The average fruit weight was obtained by dividing the total weight of marketable fruits by the number of marketable fruits. Skin elasticity was obtained by measuring two different points of the equatorial diameter of 5 fruits from each repetition with a hand penetrometer (Effegi, FT-327) (Ranatunga et al., 2008).

\section{Fruit quality analysis}

For fruit quality analysis, juice of 5 fruits taken from the $2^{\text {nd }}$ and $3^{\text {rd }}$ clusters was extracted in blender. In the fruit juice obtained; TSS amount was determined with refractometer (Reed MT-032 Brix Refractometer), titratable acidity was determined according to Cemeroğlu, (2002). Lycopene content was determined according to Fish et al. (2002), by using hexane, methanol and acetone (2:1:1). Reducing sugar content was determined with titrimetric method according to the Lane-Eynon (Cemeroğlu, 2002).

\section{Experimental design and statistical analysis}

Trial factorial randomized blocks were established with 3 repetitions according to the experimental design. Total of 1560 plants were included in each repetition with 8 plants in each repetition. Statistical analysis was carried out with SAS software package; Duncan test was applied for comparing the means (SAS, 1995).

\section{Results}

\section{Yield}

The effects of nutrient induced EC levels and rootstocks on the yield and fruit number are given in Tables 2 and 3. The total and marketable yield and the total and marketable number of fruits decreased with the increase in electrical conductivity. The highest total and marketable yield was in the $\mathrm{EC}=2 \mathrm{dS} \mathrm{m}{ }^{-1}$ treatment group, while the highest total and marketable number of fruits was obtained in the $E C=3 \mathrm{dS} \mathrm{m}^{-1}$ group. Although total and marketable yield slightly increased with rootstock use, this increase was not significant.

Percentile distribution of yield based on fruit size is illustrated in Fig. 2. As seen in this figure, $47 \%$ of the yield obtained from $E C=2 \mathrm{dS} \mathrm{m}^{-1}$ had higher fruit diameters than $67 \mathrm{~mm}$ and $43 \%$ had between $57-67 \mathrm{~mm}$ while $50 \%$ of the fruits in the EC=9 dS m${ }^{-}$ ${ }^{1}$ group had diameters between $47-57 \mathrm{~mm}, 23 \%$ between $57-67 \mathrm{~mm}$ and $10 \%$ consisting of discarded fruits. Percentile distribution of the number of fruits based on the fruit size is given in Fig. 3. About $32 \%$ of the yield obtained from $\mathrm{EC}=2 \mathrm{dS} \mathrm{m}^{-1}$ had higher fruit diameters than $67 \mathrm{~mm}$ and $43 \%$ had between $57-67 \mathrm{~mm}$ while $39 \%$ of the fruits in $\mathrm{EC}=9 \mathrm{dS} \mathrm{m}{ }^{-1}$ application had diameters between $47-57 \mathrm{~mm}$ and $29 \%$ consisted of discarded fruits. Parallel to the increase in EC levels, the ratio of discarded and smaller fruits increased. 
Table 2. The effects of nutrient induced EC levels and rootstocks on total and marketable yield and total number of fruits

\begin{tabular}{|c|c|c|c|c|c|c|c|c|c|}
\hline \multirow{2}{*}{ 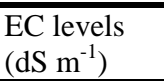 } & \multicolumn{3}{|c|}{ Total Yield $\left(\mathrm{kg} \mathrm{m}^{-2}\right)$} & \multicolumn{3}{|c|}{ Marketable yield $\left(\mathrm{kg} \mathrm{m}^{-2}\right)$} & \multicolumn{3}{|c|}{ Total fruit number $\mathrm{m}^{-2}$} \\
\hline & 2009 & 2010 & Average & 2009 & 2010 & Average & 2009 & 2010 & Average \\
\hline 2 & $8.16 \mathrm{a}$ & $9.46 \mathrm{a}$ & $8.81 \mathrm{a}$ & $8.05 \mathrm{a}$ & $9.36 \mathrm{a}$ & $8.70 \mathrm{a}$ & $88.94 \mathrm{a}$ & $94.16 \mathrm{~b}$ & $91.55 \mathrm{~b}$ \\
\hline 3 & $7.73 \mathrm{~b}$ & $9.35 \mathrm{a}$ & $8.54 \mathrm{~b}$ & $7.61 \mathrm{~b}$ & $9.23 \mathrm{a}$ & $8.42 \mathrm{~b}$ & $89.07 \mathrm{a}$ & $99.55 \mathrm{a}$ & $94.31 \mathrm{a}$ \\
\hline 5 & $5.21 \mathrm{c}$ & $7.24 \mathrm{~b}$ & $6.23 \mathrm{c}$ & $5.02 \mathrm{c}$ & $7.02 \mathrm{~b}$ & $6.02 \mathrm{c}$ & $80.66 \mathrm{~b}$ & $98.85 \mathrm{a}$ & $89.75 b$ \\
\hline 7 & $4.12 \mathrm{~d}$ & $5.19 \mathrm{c}$ & $4.65 \mathrm{~d}$ & $3.81 \mathrm{~d}$ & $4.88 \mathrm{c}$ & $4.34 \mathrm{~d}$ & $80.88 \mathrm{~b}$ & $89.13 \mathrm{c}$ & $85.00 \mathrm{c}$ \\
\hline 9 & $2.93 \mathrm{e}$ & $4.16 \mathrm{~d}$ & $3.55 \mathrm{e}$ & $2.57 \mathrm{e}$ & $3.80 \mathrm{~d}$ & $3.18 \mathrm{e}$ & $67.64 \mathrm{c}$ & $87.37 \mathrm{c}$ & $77.51 \mathrm{~d}$ \\
\hline Significance & $* *$ & $* *$ & $* *$ & $* *$ & $* *$ & $* *$ & $* *$ & $* *$ & $* *$ \\
\hline \multicolumn{10}{|l|}{ Rootstocks } \\
\hline Nongrafted & $5.29 \mathrm{~b}$ & 6.97 & 6.13 & 5.14 & 6.75 & 5.95 & $73.11 \mathrm{~b}$ & 93.36 & $83.24 \mathrm{c}$ \\
\hline Selfgrafted & $5.56 \mathrm{ab}$ & 7.02 & 6.29 & 5.32 & 6.80 & 6.06 & $83.36 \mathrm{a}$ & 94.24 & $88.80 \mathrm{a}$ \\
\hline Heman & $5.69 \mathrm{ab}$ & 7.34 & 6.52 & 5.50 & 7.13 & 6.32 & $79.48 \mathrm{a}$ & 94.91 & $87.19 \mathrm{ab}$ \\
\hline Resistar & $5.63 \mathrm{ab}$ & 7.14 & 6.38 & 5.36 & 6.91 & 6.14 & $82.07 \mathrm{a}$ & 95.53 & $88.80 \mathrm{a}$ \\
\hline Unifort & $5.30 \mathrm{~b}$ & 7.21 & 6.25 & 5.13 & 6.98 & 6.06 & $73.48 \mathrm{~b}$ & 94.53 & $84.01 \mathrm{bc}$ \\
\hline Beaufort & $5.63 \mathrm{ab}$ & 6.97 & 6.30 & 5.39 & 6.76 & 6.07 & $83.83 \mathrm{a}$ & 92.37 & $88.10 \mathrm{a}$ \\
\hline Maxifort & $5.82 \mathrm{a}$ & 7.33 & 6.57 & 5.56 & 7.12 & 6.34 & $84.40 \mathrm{a}$ & 95.33 & $89.86 \mathrm{a}$ \\
\hline Kemerit & $5.82 \mathrm{a}$ & 7.04 & 6.43 & 5.65 & 6.82 & 6.23 & $81.01 \mathrm{a}$ & 93.41 & $87.21 \mathrm{ab}$ \\
\hline Yedi RZ & $5.80 \mathrm{a}$ & 7.06 & 6.43 & 5.56 & 6.84 & 6.20 & $84.86 \mathrm{a}$ & 90.91 & $87.89 \mathrm{ab}$ \\
\hline Spirit & $5.72 \mathrm{ab}$ & 7.06 & 6.39 & 5.48 & 6.81 & 6.15 & $83.75 \mathrm{a}$ & 95.28 & $89.52 \mathrm{a}$ \\
\hline Kingkong & $5.43 \mathrm{ab}$ & 6.95 & 6.19 & 5.21 & 6.74 & 5.97 & $80.44 \mathrm{a}$ & 92.12 & $86.28 \mathrm{a}-\mathrm{c}$ \\
\hline Toro & $5.68 \mathrm{ab}$ & 6.81 & 6.25 & 5.46 & 6.55 & 6.01 & $84.00 \mathrm{a}$ & 93.80 & $88.90 \mathrm{a}$ \\
\hline Body & $5.84 \mathrm{a}$ & 7.13 & 6.48 & 5.59 & 6.91 & 6.25 & $84.90 \mathrm{a}$ & 93.78 & $89.34 \mathrm{a}$ \\
\hline Significance & $*$ & ns & ns & ns & ns & ns & ** & ns & $* *$ \\
\hline
\end{tabular}

Different letters indicate significant differences at $\mathrm{P}<0.05$ by Duncan test $(\mathrm{n}=3)$.

$*$,** represent $\mathrm{P}<0.05$ and $\mathrm{P}<0.01$, respectively.

ns, not significant.

Table 3. The effects of nutrient induced EC levels and rootstocks on the number of fruits, average fruit weight and fruit diameter

\begin{tabular}{|c|c|c|c|c|c|c|c|c|c|}
\hline \multirow[t]{2}{*}{$\begin{array}{l}\text { EC levels } \\
\left(\mathrm{dS} \mathrm{m}^{-1}\right)\end{array}$} & \multicolumn{3}{|c|}{$\begin{array}{l}\text { Marketable fruit number } \\
\left.\text { (number } \mathrm{m}^{-2}\right)\end{array}$} & \multicolumn{3}{|c|}{ Average fruit weight $(\mathrm{g})$} & \multicolumn{3}{|c|}{ Fruit diameter $(\mathrm{mm})$} \\
\hline & 2009 & 2010 & Average & 2009 & 2010 & Average & 2009 & 2010 & Average \\
\hline 2 & $81.36 \mathrm{a}$ & $86.98 \mathrm{~b}$ & $84.17 \mathrm{~b}$ & $98.96 \mathrm{a}$ & $107.75 \mathrm{a}$ & $103.35 \mathrm{a}$ & $62.02 \mathrm{a}$ & $63.39 \mathrm{a}$ & $62.71 \mathrm{a}$ \\
\hline 3 & $81.35 \mathrm{a}$ & $91.60 \mathrm{a}$ & $86.47 \mathrm{a}$ & $93.61 \mathrm{~b}$ & $100.75 \mathrm{~b}$ & $97.18 \mathrm{~b}$ & $60.31 \mathrm{~b}$ & $63.63 \mathrm{a}$ & $61.97 \mathrm{~b}$ \\
\hline 5 & $68.39 \mathrm{~b}$ & $84.43 \mathrm{~b}$ & $76.40 \mathrm{c}$ & $73.42 \mathrm{c}$ & $83.10 \mathrm{c}$ & $78.26 \mathrm{c}$ & $55.16 \mathrm{c}$ & $59.19 \mathrm{~b}$ & $57.18 \mathrm{c}$ \\
\hline 7 & $61.31 \mathrm{c}$ & $69.96 \mathrm{c}$ & $65.64 \mathrm{~d}$ & $61.94 \mathrm{~d}$ & $69.59 \mathrm{~d}$ & $65.77 \mathrm{~d}$ & $51.36 \mathrm{~d}$ & $57.19 \mathrm{c}$ & $54.27 \mathrm{~d}$ \\
\hline 9 & $47.16 \mathrm{~d}$ & $63.52 \mathrm{~d}$ & $55.34 \mathrm{e}$ & $54.38 \mathrm{e}$ & $59.64 \mathrm{e}$ & $57.01 \mathrm{e}$ & $48.90 \mathrm{e}$ & $52.48 \mathrm{~d}$ & $50.69 \mathrm{e}$ \\
\hline Significance & $* *$ & $* *$ & $* *$ & $* *$ & $* *$ & $* *$ & $* *$ & $* *$ & $* *$ \\
\hline \multicolumn{10}{|l|}{ Rootstocks } \\
\hline Nongrafted & $63.63 \mathrm{c}$ & 78.58 & 71.10 & 78.64 & $82.95 \mathrm{~b}-\mathrm{d}$ & $80.80 \mathrm{a}-\mathrm{c}$ & 55.86 & 58.17 & 57.02 \\
\hline Selfgrafted & $68.32 \mathrm{a}-\mathrm{c}$ & 79.90 & 74.11 & 74.52 & $83.05 \mathrm{~b}-\mathrm{d}$ & 78.79 bc & 54.80 & 58.77 & 56.79 \\
\hline Heman & $67.36 \mathrm{a}-\mathrm{c}$ & 80.81 & 74.09 & 78.00 & $85.94 \mathrm{ab}$ & $81.97 \mathrm{a}$ & 55.81 & 59.85 & 57.83 \\
\hline Resistar & $66.74 \mathrm{a}-\mathrm{c}$ & 80.38 & 73.56 & 75.72 & $83.82 \mathrm{a}-\mathrm{d}$ & $79.77 \mathrm{a}-\mathrm{c}$ & 55.36 & 59.86 & 57.61 \\
\hline Unifort & $64.27 \mathrm{bc}$ & 80.05 & 72.16 & 77.04 & $85.58 \mathrm{a}-\mathrm{c}$ & $81.31 \mathrm{ab}$ & 54.88 & 59.33 & 57.11 \\
\hline Beaufort & $68.62 \mathrm{a}-\mathrm{c}$ & 78.05 & 73.33 & 74.97 & $84.33 \mathrm{a}-\mathrm{d}$ & 79.65 a-c & 55.55 & 59.29 & 57.42 \\
\hline Maxifort & $69.56 \mathrm{a}$ & 81.11 & 75.33 & 76.97 & $85.72 \mathrm{ab}$ & $81.34 \mathrm{ab}$ & 56.18 & 58.65 & 57.42 \\
\hline Kemerit & $69.60 \mathrm{a}$ & 80.15 & 74.88 & 77.11 & $82.48 \mathrm{~cd}$ & $79.80 \mathrm{a}-\mathrm{c}$ & 55.74 & 58.40 & 57.07 \\
\hline Yedi RZ & $69.26 \mathrm{ab}$ & 77.33 & 73.30 & 77.57 & $86.65 \mathrm{a}$ & $82.11 \mathrm{a}$ & 56.03 & 59.29 & 57.66 \\
\hline Spirit & $69.58 \mathrm{a}$ & 78.22 & 73.90 & 75.64 & 84.44 a-d & $80.04 \mathrm{a}-\mathrm{c}$ & 55.39 & 60.55 & 57.97 \\
\hline Kingkong & $65.31 \mathrm{a}-\mathrm{c}$ & 79.18 & 72.24 & 76.53 & $82.27 \mathrm{~d}$ & 79.39 bc & 55.99 & 58.83 & 57.41 \\
\hline Toro & $70.12 \mathrm{a}$ & 77.44 & 73.78 & 74.67 & $82.24 \mathrm{~d}$ & $78.45 \mathrm{c}$ & 55.27 & 59.02 & 57.14 \\
\hline Body & $70.49 \mathrm{a}$ & 79.65 & 75.07 & 76.65 & $84.68 \mathrm{a}-\mathrm{d}$ & $80.66 \mathrm{a}-\mathrm{c}$ & 55.29 & 59.29 & 57.29 \\
\hline Significance & $*$ & ns & ns & ns & $* *$ & $*$ & ns & ns & ns \\
\hline
\end{tabular}




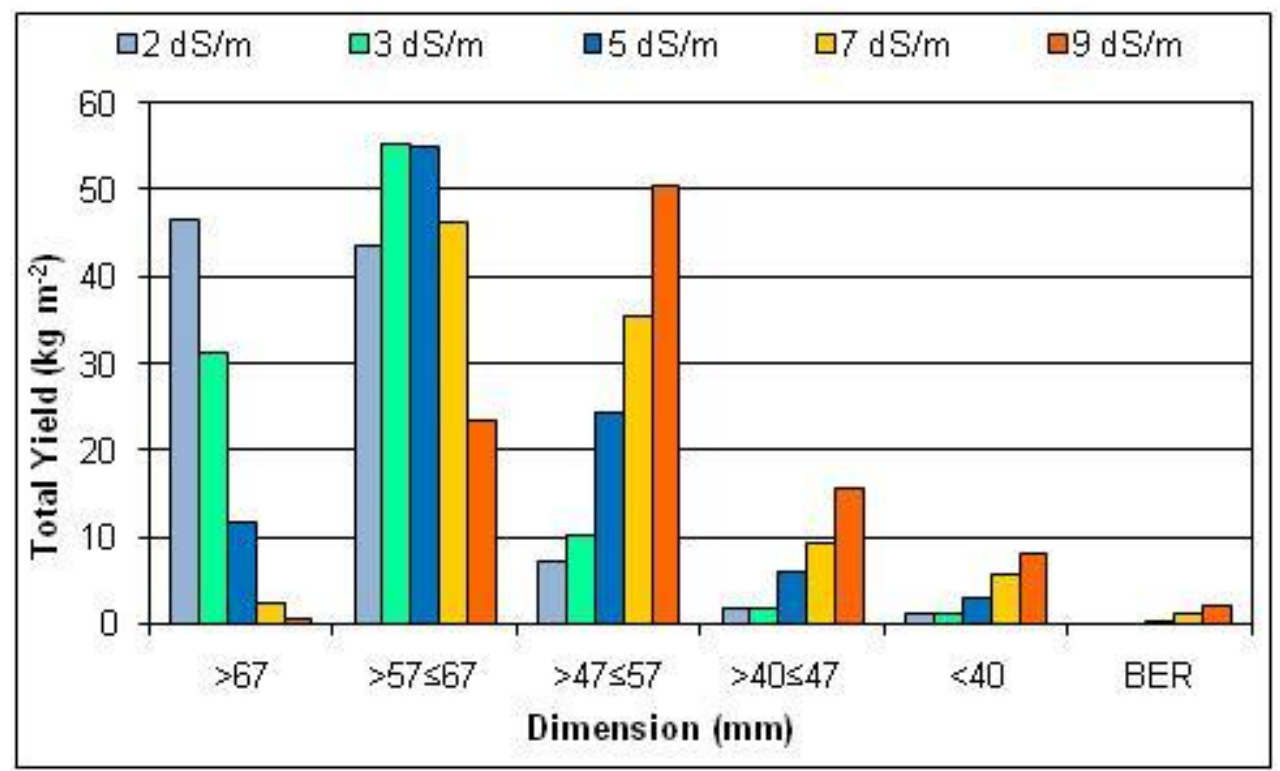

Figure 2. Percentile distribution of total yield obtained from nutrient induced salinity levels based on fruit size

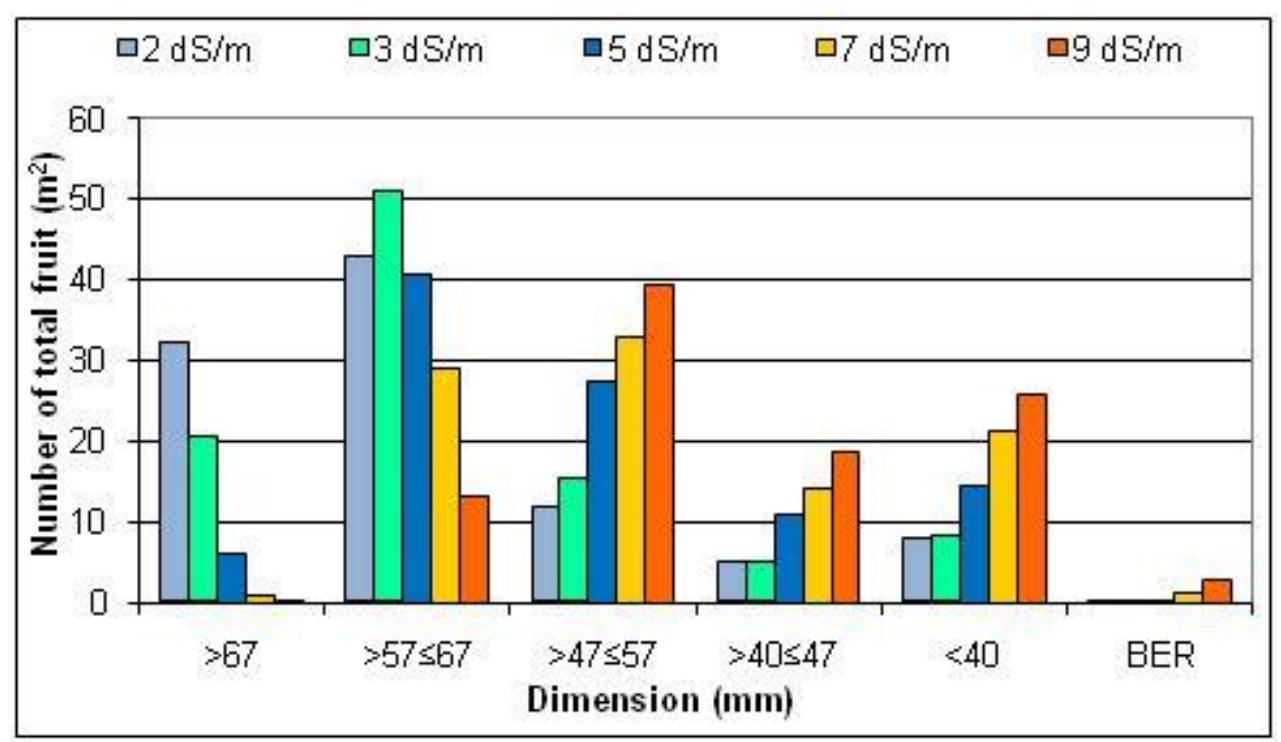

Figure 3. Percentile distribution of total yield obtained from nutrient induced salinity levels based on the number of fruits

\section{Fruit properties}

The increase in electrical conductivity had a negative effect on the average fruit weight, fruit diameter and pericarp thickness; however skin firmness increased. The highest average fruit weight, fruit diameter and pericarp thickness was obtained from $E C=2 \mathrm{dS} \mathrm{m}^{-1}$ application. In general, the effect of rootstocks on the fruit properties was not significant (Tables 3, 4). 
Table 4. The effect of nutrient induced EC levels and rootstocks on pericarp thickness, skin firmness and the amount of TSS

\begin{tabular}{|c|c|c|c|c|c|c|c|c|c|}
\hline \multirow[t]{2}{*}{ 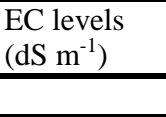 } & \multicolumn{3}{|c|}{ Pericarp thickness (mm) } & \multicolumn{3}{|c|}{ Firmness $\left(\left(\mathrm{kg} \mathrm{cm}^{-2}\right)\right.$} & \multicolumn{3}{|c|}{ TSS (Brix) } \\
\hline & 2009 & 2010 & Average & 2009 & 2010 & Average & 2009 & 2010 & Average \\
\hline 2 & $7.36 \mathrm{a}$ & $8.34 \mathrm{a}$ & $7.85 \mathrm{a}$ & $3.79 \mathrm{~d}$ & $3.27 \mathrm{c}$ & $3.53 \mathrm{~b}$ & $4.61 \mathrm{e}$ & $4.34 \mathrm{e}$ & $4.47 \mathrm{e}$ \\
\hline 3 & $6.96 \mathrm{~b}$ & $7.74 \mathrm{~b}$ & $7.35 \mathrm{~b}$ & $3.44 \mathrm{e}$ & $3.74 \mathrm{~b}$ & $3.59 \mathrm{~b}$ & $4.94 \mathrm{~d}$ & $5.09 \mathrm{~d}$ & $5.02 \mathrm{~d}$ \\
\hline 5 & $6.91 \mathrm{~b}$ & $7.50 \mathrm{~b}$ & $7.21 \mathrm{~b}$ & $4.09 \mathrm{c}$ & $4.17 \mathrm{a}$ & $4.13 \mathrm{a}$ & $5.34 \mathrm{c}$ & $5.29 \mathrm{c}$ & $5.32 \mathrm{c}$ \\
\hline 7 & $6.75 \mathrm{~b}$ & $6.03 \mathrm{c}$ & $6.39 c$ & $4.44 \mathrm{~b}$ & $3.99 \mathrm{a}$ & $4.22 \mathrm{a}$ & $5.66 \mathrm{~b}$ & $5.99 \mathrm{~b}$ & $5.83 \mathrm{~b}$ \\
\hline 9 & $6.37 \mathrm{c}$ & $4.94 \mathrm{~d}$ & $5.65 \mathrm{~d}$ & $4.70 \mathrm{a}$ & $3.69 \mathrm{~b}$ & $4.20 \mathrm{a}$ & $6.21 \mathrm{a}$ & $6.78 \mathrm{a}$ & $6.50 \mathrm{a}$ \\
\hline Significance & $* *$ & $* *$ & $* *$ & $* *$ & $* *$ & & $* *$ & $* *$ & $* *$ \\
\hline \multicolumn{10}{|l|}{ Rootstocks } \\
\hline Nongrafted & 6.86 & 6.90 & 6.88 & 4.20 & 3.66 & 3.93 & 5.06 & 5.53 & 5.29 \\
\hline Selfgrafted & 6.73 & 6.81 & 6.77 & 4.16 & 3.91 & 4.04 & 5.41 & 5.67 & 5.54 \\
\hline Heman & 6.75 & 6.84 & 6.80 & 4.12 & 3.71 & 3.92 & 5.42 & 5.56 & 5.49 \\
\hline Resistar & 6.73 & 6.86 & 6.80 & 4.21 & 3.68 & 3.95 & 5.19 & 5.44 & 5.32 \\
\hline Unifort & 6.92 & 6.98 & 6.95 & 4.06 & 3.80 & 3.93 & 5.48 & 5.49 & 5.49 \\
\hline Beaufort & 6.87 & 7.02 & 6.94 & 4.11 & 3.82 & 3.96 & 5.44 & 5.44 & 5.44 \\
\hline Maxifort & 6.92 & 6.96 & 6.94 & 4.01 & 3.78 & 3.90 & 5.44 & 5.51 & 5.48 \\
\hline Kemerit & 6.95 & 7.10 & 7.02 & 3.97 & 3.91 & 3.94 & 5.25 & 5.38 & 5.32 \\
\hline Yedi RZ & 7.04 & 6.83 & 6.93 & 3.95 & 3.90 & 3.92 & 5.39 & 5.49 & 5.44 \\
\hline Spirit & 6.92 & 6.99 & 6.95 & 4.09 & 3.84 & 3.97 & 5.42 & 5.56 & 5.49 \\
\hline Kingkong & 6.96 & 6.79 & 6.87 & 4.12 & 3.74 & 3.94 & 5.41 & 5.55 & 5.48 \\
\hline Toro & 6.91 & 6.86 & 6.89 & 4.26 & 3.75 & 4.00 & 5.47 & 5.45 & 5.46 \\
\hline Body & 6.74 & 6.88 & 6.81 & 3.94 & 3.57 & 3.76 & 5.22 & 5.39 & 5.31 \\
\hline Significance & ns & ns & ns & $\mathrm{ns}$ & $\mathrm{ns}$ & $\mathrm{ns}$ & öd & öd & ns \\
\hline
\end{tabular}

Different letters indicate significant differences at $\mathrm{P}<0.05$ by Duncan test $(\mathrm{n}=3)$.

*** represent $\mathrm{P}<0.05$ and $\mathrm{P}<0.01$, respectively.

ns, not significant.

Table 5. The effect of nutrient induced EC levels and rootstocks on lycopene, titratable acidity and reducing sugar contents

\begin{tabular}{|c|c|c|c|c|c|c|c|c|c|}
\hline \multirow[t]{2}{*}{ 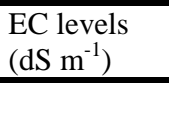 } & \multicolumn{3}{|c|}{ Lycopene (mg kg ${ }^{-1}$ ) } & \multicolumn{3}{|c|}{$\begin{array}{c}\text { Titratable acidity } \\
\left(\mathrm{g} \text { citric acid } 100 \mathrm{ml}^{-1}\right)\end{array}$} & \multicolumn{3}{|c|}{ Reducing sugar $(\%)$} \\
\hline & 2009 & 2010 & Average & 2009 & 2010 & Average & 2009 & 2010 & Average \\
\hline 2 & $72.39 \mathrm{c}$ & $47.98 \mathrm{~d}$ & $60.19 d$ & $0.28 \mathrm{e}$ & $0.26 \mathrm{e}$ & $0.27 \mathrm{e}$ & $2.68 \mathrm{~b}$ & $2.34 \mathrm{~d}$ & $2.51 \mathrm{~d}$ \\
\hline 3 & $81.26 \mathrm{~b}$ & $65.30 \mathrm{c}$ & $73.28 \mathrm{c}$ & $0.30 \mathrm{~d}$ & $0.37 \mathrm{~d}$ & $0.34 \mathrm{~d}$ & $3.00 \mathrm{a}$ & $2.59 \mathrm{c}$ & $2.80 \mathrm{c}$ \\
\hline 5 & $94.54 \mathrm{a}$ & $74.46 \mathrm{~b}$ & $84.50 \mathrm{~b}$ & $0.35 \mathrm{c}$ & $0.41 \mathrm{c}$ & $0.38 \mathrm{c}$ & $3.01 \mathrm{a}$ & $2.76 \mathrm{c}$ & $2.88 \mathrm{c}$ \\
\hline 7 & $96.10 \mathrm{a}$ & $95.95 \mathrm{a}$ & $96.02 \mathrm{a}$ & $0.40 \mathrm{~b}$ & $0.45 \mathrm{~b}$ & $0.43 \mathrm{~b}$ & $3.09 \mathrm{a}$ & $3.13 \mathrm{~b}$ & $3.11 \mathrm{~b}$ \\
\hline 9 & $83.97 \mathrm{~b}$ & $100.60 \mathrm{a}$ & $92.29 \mathrm{a}$ & $0.51 \mathrm{a}$ & $0.54 \mathrm{a}$ & $0.53 \mathrm{a}$ & $3.08 \mathrm{a}$ & $3.57 \mathrm{a}$ & $3.32 \mathrm{a}$ \\
\hline Significance & $* *$ & $* *$ & $* *$ & ** & $* *$ & $* *$ & $* *$ & $* *$ & $* *$ \\
\hline \multicolumn{10}{|l|}{ Rootstocks } \\
\hline Nongrafted & 88.29 & 75.55 & 81.92 & $0.37 \mathrm{a}-\mathrm{c}$ & 0.41 & $0.39 \mathrm{a}-\mathrm{c}$ & 2.86 & 3.04 & 2.95 \\
\hline Selfgrafted & 84.20 & 80.50 & 82.35 & $0.37 \mathrm{a}-\mathrm{c}$ & 0.42 & $0.40 \mathrm{ab}$ & 2.97 & 2.93 & 2.95 \\
\hline Heman & 94.31 & 75.03 & 84.67 & $0.39 \mathrm{ab}$ & 0.42 & $0.41 \mathrm{ab}$ & 3.05 & 2.80 & 2.93 \\
\hline Resistar & 77.99 & 72.23 & 75.11 & $0.40 \mathrm{a}$ & 0.42 & $0.41 \mathrm{a}$ & 2.85 & 2.73 & 2.79 \\
\hline Unifort & 84.93 & 75.68 & 80.31 & $0.37 \mathrm{a}-\mathrm{c}$ & 0.41 & $0.39 \mathrm{a}-\mathrm{c}$ & 2.96 & 2.83 & 2.90 \\
\hline Beaufort & 82.70 & 76.97 & 79.84 & $0.38 \mathrm{ab}$ & 0.39 & 0.39 a-c & 2.92 & 2.93 & 2.92 \\
\hline Maxifort & 79.24 & 77.14 & 78.19 & $0.39 \mathrm{ab}$ & 0.41 & $0.40 \mathrm{ab}$ & 2.87 & 2.74 & 2.80 \\
\hline Kemerit & 89.54 & 75.43 & 82.48 & $0.35 \mathrm{~b}-\mathrm{d}$ & 0.40 & $0.38 \mathrm{bd}$ & 2.94 & 2.96 & 2.95 \\
\hline Yedi RZ & 85.06 & 75.97 & 80.51 & $0.36 \mathrm{~b}-\mathrm{d}$ & 0.42 & $0.39 \mathrm{a}-\mathrm{c}$ & 2.99 & 2.84 & 2.92 \\
\hline Spirit & 86.04 & 78.23 & 82.13 & $0.35 \mathrm{~b}-\mathrm{d}$ & 0.41 & $0.38 \mathrm{bc}$ & 3.00 & 2.95 & 2.97 \\
\hline Kingkong & 85.82 & 80.20 & 83.01 & $0.34 \mathrm{~cd}$ & 0.39 & $0.37 \mathrm{~cd}$ & 3.09 & 2.77 & 2.93 \\
\hline Toro & 85.25 & 78.89 & 82.07 & $0.37 \mathrm{a}-\mathrm{c}$ & 0.39 & $0.38 \mathrm{~b}-\mathrm{d}$ & 3.09 & 2.82 & 2.95 \\
\hline Body & 90.12 & 77.33 & 83.73 & $0.33 \mathrm{~d}$ & 0.37 & $0.35 \mathrm{~d}$ & 3.04 & 3.06 & 3.05 \\
\hline Significance & ns & ns & ns & $* *$ & ns & $* *$ & ns & ns & ns \\
\hline
\end{tabular}

Different letters indicate significant differences at $\mathrm{P}<0.05$ by Duncan test $(\mathrm{n}=3)$. 
*** represent $\mathrm{P}<0.05$ and $\mathrm{P}<0.01$, respectively.

ns, not significant.

\section{Quality}

High electrical conductivity caused increases in TSS, titratable acidity, lycopene and reducing sugar quality levels. The highest values were obtained in $\mathrm{EC}=9 \mathrm{dS} \mathrm{m}^{-1}$, while the lowest values were in the $\mathrm{EC}=2 \mathrm{dS} \mathrm{m} \mathrm{m}^{-1}$ application. The effect of rootstocks on the quality was not significant (Tables 4,5$)$.

\section{Discussion}

The results showed that the increasing nutrient induced EC levels reduced the total and marketable fruit yield, the number of fruits, the average fruit weight, fruit diameter and pericarp thickness, while skin firmness is increased. Sonneveld and Welles (1988) reported that high EC-values in the root environment of tomato lowered fruit yield. Many studies have confirmed that increased salt levels in nutrient solution leads to decreased yield in tomatoes (Krauss et al., 2006; Yokaş et al., 2008; Flores et al., 2010; Semiz and Suarez, 2015), cucumber (Huang et al., 2009) and water melon (Colla et al., 2006). This overall decrease in yield may be associated with the reduction in the number of fruits, fruit diameter and fruit weight induced by the increase in EC levels. We also confirmed that the number of total and marketable fruits was decreased by increasing the EC levels in our study. Similarly, decreases in the number of fruits were reported by Huang et al. (2009) in cucumber and by Amor et al. (2001) and Tüzel et al. (2001) in tomatoes by increasing the salinity. Although Colla et al. (2006) for water melon and Eltez et al. (2002) for tomatoes reported that increased salinity had no effect on the number of fruits.

The average fruit weight, fruit diameter and pericarp thickness were also negatively affected by high EC levels. About $90 \%$ of the yield and $75 \%$ of the number of the fruits obtained from $E C=2 \mathrm{dS} \mathrm{m}^{-1}$ had larger fruit diameters than $57 \mathrm{~mm}$, while $76 \%$ of the yield and $87 \%$ of the number of the fruits obtained from $\mathrm{EC}=9 \mathrm{dS} \mathrm{m}{ }^{-1}$ application had fruits with diameters smaller than $57 \mathrm{~mm}$. The size of the fruits decreased as the EC levels increased (Figs. 2 and 3). Our results are compatible with previous studies on different genera that reported a decrease in the average focused weight (Amor et al., 2001; Colla et al., 2006; Krauss et al., 2006; Yokaş et al., 2008; Huang et al., 2009 and Savvas et al., 2011), fruit diameter (Cramer et al., 2001; Amor et al., 2001; Magan et al., 2008) and pericarp thickness (Morales-Garcia et al., 2010). Skin firmness increased along with the increased in EC levels. Munns and Tester (2008), reported that plants have evolved mechanisms to tolerate the low soil water potential caused by salinity and drought.

Fruit quality parameters were positively affected by salinity; as EC levels increased, fruit quality is increased. According to previous studies, with increased salinity levels in the nutrient solution, the amounts of TSS (Amor et al., 2001; Tüzel et al., 2001; Eltez et al., 2002; Krauss et al., 2006; Öztekin, 2009), lycopene (Wu et al., 2004; Krauss et al., 2006; Giannakoula and Ilias, 2013), titratable acidity (Tüzel et al., 2001; Eltez et al., 2002; Krauss et al., 2006; Öztekin, 2009; Savvas et al., 2011) and reducing sugar contents increased (Mizrahi et al., 1982; Amor et al., 2001). Water deficit is the primary effect of salt stress due to lowered water potential of the soil solution and restricted root water uptake (Ferreira-Silva et al., 2008). Osmotic adjustment in plants subjected to salt 
stress can occur by the accumulation of high concentrations of either inorganic ions or low molecular weight organic solutes (Ashraf and Harris, 2004).

Comparing the averages of the rootstocks to the controls, it was seen that rootstock use caused slight increases in the total yield, marketable yield, total number of fruits and the number of marketable fruits. It was reported that grafting increases the total yield in tomatoes (Gajc-Wolska et al., 2010; Turhan et al., 2011; Sanches-Rodriguez et al., 2012; Voutsela et al., 2012; Öztekin et al., 2013; Semiz and Suarez, 2015), in eggplant (Khah, 2011) and in watermelon (Colla et al., 2006). Similarly, it was reported that marketable yield increased in tomatoes with rootstock use (Geboloğlu, 2011; Öztekin et al., 2013; Di Gioia et al., 2013). There are conflicting results concerning the effects of rootstocks on the number of fruits. According to the results obtained by Öztekin et al. (2013), there were no difference between the numbers of fruits in self-grafted plants and the plants grafted on Beaufort and Maxifort rootstocks. Kacjan-Marsic and Osvald (2004) grafted Belle and Monroe cultivars on two different types of rootstocks and compared them with non-grafted control plants; Belle cultivar formed a lower number of fruits on both of the rootstocks compared to the control, while Monroe cultivar produced the highest yield when grafted onto Beaufort rootstock.

The effects of rootstocks on the average fruit weight, fruit diameter and fruit firmness were not significant. Some studies have reported that the average fruit weight increases with rootstock use (Pogonyi et al., 2005; Geboloğlu et al., 2011; Turhan et al., 2011; Öztekin et al., 2013), while some have reported that rootstocks have no effect on the fruit weight (Savvas et al., 2011). Qaryouti et al. (2007) found that grafting had no effect on the fruit size, although fruit firmness was slightly improved.

The effect of rootstocks on the amount of TSS was found not statistically significant (Colla et al., 2006; Huang et al., 2009; Geboloğlu et al., 2011; Savvas et al., 2011; Öztekin et al., 2013; Di Gioia et al., 2013), although some researchers have observed that soluble solid content was lower in grafted plants compared to non-grafted plants (Pogonyi et al., 2005; Turhan et al., 2011; Al-Harbi et al., 2016). The effect of rootstocks on titratable acidity has been found to be significant (Öztekin et al., 2013). However, in studies on eggplant (Khah, 2011) and tomatoes (Pogonyi et al., 2005; Geboloğlu et al., 2011; Turhan et al., 2011; Savvas et al., 2011), found no effect of grafting on the titratable acidity. The effect of rootstocks on lycopene content has also been found to be insignificant (Vrcek et al., 2011). Mohammed et al. (2009) reported that grafting reduced the lycopene content. other reports showed no effect of rootstocks on reducing sugar content. On the other hand Gajc-Wolska et al. (2010) and Turhan et al. (2011) found decreasing sugar contents in fruits of grafted tomatoes.

\section{Conclusion}

Nutrient induced high EC applications had negative influence on the number of fruits, fruit diameter, and pericarp thickness; consequently, overall yield has decreased. In spite of this decrease in yield, important quality parameters including soluble solid contents, lycopene, titratable acidity and reducing sugars increased. We concluded that EC 7 and $9 \mathrm{dS} \mathrm{m}{ }^{-1}$ applications were not suitable for economic farming, due to low yield and the high percentage of discarded fruits. It is estimated that $\mathrm{EC}=5 \mathrm{dS} \mathrm{m}{ }^{-1}$ will be a better application in the case of demand for a fruit with a good market price and high intrinsic quality. It was determined that the yield was higher compared to control 
group depending on the genotype of the rootstocks used, although rootstocks had no statistically significant effect on the other properties examined.

Acknowledgements. This research was supported by Harran University Scientific Research Fund (Contract No.:1164).

\section{REFERENCES}

[1] Al-Harbi, A., Hejazi, A., Al-Omran, A. (2016): Response of grafted tomato (Solanum lycopersicon L.) to abiotic stress in Saudi Arabia. - Saudi Journal of Biological Sciences (In press) (http://dx.doi.org/10.1016/j.sjbs.2016.01.005)

[2] Aloni, B., Cohen, R., Karni, L., Aktas, H., Edelstein, M. (2010): Hormonal signaling in rootstock-scion interactions. - Scientia Horticulturae 127: 119-126.

[3] Ashraf, M., Harris, P.J.C. (2004): Potential biochemical indicators of salinity tolerance in plants. - Plant Science 166: 3-16.

[4] Cemeroğlu, B. (1992): Meyve ve sebze işleme endüstrisinde temel analiz metotları (Basic analysis methods in fruit and vegetable processing industry). - Biltav Üniversite Kitapları Serisi (University Book Series,. Biltav edition) No: 02-2. Ankara/Turkey, p. 381.

[5] Colla, G., Rouphael, Y., Cardarelli, M. (2006): Effect of salinity on yield, fruit quality, leaf gas exchange, and mineral composition of grafted watermelon plants. - HortScience 41(3): 622-627.

[6] Cramer, M.D., Oberholzer, J.A., Combrink N.J.J. (2001): The Effect of Supplementation of Root Zone Dissolved Inorganic Carbon on Fruit Yield and Quality of Tomatoes $(\mathrm{Cv}$ 'Daniella') Grown with Salinity. - Scientia Horticulturae 89: 269-289.

[7] Cuartero, J., Bolarin, M.C., Asins, M.J., Moreno, V. (2006): Increasing salt tolerance in the tomato. - Journal of Experimental Botany 57(5): 1045-1058.

[8] Cuartero, J., Fernandez-Munoz, R. (1999): Tomato and salinity. - Scientia Horticulturae 78: 83-125.

[9] Davis, A.R., Perkins-Veazie, P., Hassel, R., Levi, A., King, S.R., Zhang, X. (2008): Grafting effects on vegetable quality. - Hortsience 43(6): 1670-1672.

[10] Di Gioia, F., Signore, A., Serio, F., Santamaria, P. (2013): Grafting improves tomato salinity tolerance through sodium partitioning within the shoot. - Hort. Sci. 48: 855-862.

[11] Dorais, M., Ehret, D.L., Papadopoulos, A.P. (2008): Tomato (Solanum lycopersicum) health components: from the seed to the consumer. - Phytochem Rev. 7: 231-250.

[12] Eltez, R.Z., Tüzel, Y., Gül, A., Tüzel, İ.H., Duyar, H. (2002): Effects of different EC levels of nutrient solution on greenhouse tomato growing. - Acta Horticulturae 573: 443448.

[13] Eraslan, F., Güneş, A., İnal, A., Çiçek, N., Alpaslan, M. (2008): Gübrelerden kaynaklanan tuzluluğun domates ve biber bitkisinde bazı fizyolojik özellikler ve mineral beslenme üzerine etkisi (Effect of fertilizer induced salinity on some physiological parameters and mineral nutrition of tomato and pepper plant). - 4. Ulusal Bitki Besleme ve Gübre Kongresi (4th National Congress of Plant Nutrition and Fertilizer), Konya/Turkey, pp. 641-649.

[14] Ferreira-Silva, S.L., J.A.G. Silveira, E.L. Voigt, L.S.P. Soares, Viégas, R.A. (2008): Changes in physiological indicators associated with salt tolerance in two contrasting cashew rootstocks. - Braz. J. Plant Physiol. 20(1): 51-59.

[15] Fish, W. W., Perkins-Veazie, P., Collins, J. K. (2002): A quantitative assay for lycopene that utilizes reduced volumes of organic solvents. - Journal of Food Composition and Analysis 15: 309-317.

[16] Flores, F.B., Sanchez-Bel, P., Estan, M.T., Martinez-Rodriguez, M.M., Moyano, E., Morales, B., Campos, J.F., Garcia-Abellan, J.O., Egea, M.I., Fernandez-Garcia, N., 
Romojaro, F., Bolorin, M.C. (2010): The Efectiveness of grafting to improve tomato fruit quality. - Scientia Horticulturae 125: 211-217.

[17] Geboloğlu, N., Yılmaz, E., Çakmak, P., Aydın, M., Kasap, Y. (2011): Determining of the yield, quality and nutrient content of tomatoes grafted on different rootstocks in soilless culture. - Scientific Research and Essays 6(10): 2147-2153.

[18] Giannakoula, A.E., Ilias, I.F. (2013): The effect of water stres and salinity on growth and physiology of tomato (Lycopersicon esculentun Mill.). - Archive of Biological Sciences, Belgrade 65(2): 611-620.

[19] Huang, Y., Tang, R., Cao, Q., Bie, Z. (2009): Improving the fruit yield and quality of cucumber by grafting onto the salt tolerant rootstock under $\mathrm{NaCl}$ stres. - Scientia Horticulturae 122: 26-31.

[20] Kacjan Maršić, N., Osvald, J. (2004): The Influence of grafting on yield of two tomato cultivars (Lycopersicon esculentum Mill.) grown in a plastic house. - Acta Agriculturae Slovenica 83(2): 243-249.

[21] Khah, E.M. (2011): Effect of grafting on growth, performance and yield of aubergine (Solanum melongena L.) in greenhouse and open-field. - International Journal of Plant Production 5(4): 359-366.

[22] Krauss, S., Schnitzler, W.H., Grassman, J., Woitke, M. (2007): Fruit quality characteristics of tomatoes at different EC values in a simplified recirculating soilless system. - Acta Horticulturae 747: 457-463.

[23] Maas, E.V. (1993): "Testing crops for salinity tolerance." - Proceeding of the Workshop on Adaptation of Plants to Soil Stresses, August 1-4,1993. University of Nebraska. Lincoln, Nebraska.

[24] Magan, J. J., Gallardo, M., Thompson, R. B., Lorenzo, P. (2008): Effects of salinity on fruit yield and quality of tomato grown in soilless culture in greenhouses in Mediterranean climatic conditions. - Agricultural Water Managment 95: 1041-1055.

[25] Mitchell, J.P., Shennan, C., Grattan, S.R., May, D.M. (1991): Tomato fruit yields and quality under water deficit and salinity. - J. Amer. Soc. Hort. Sci. 116(2): 215-221.

[26] Mizrahi, Y. (1982): Effect of salinity on tomato fruit ripening. - Plant Physiol. 69: 966970.

[27] Mohammed, S.M.T., Humidan, M., Boras, M., Abdalla, O.A. (2009): Effect of grafting tomato on different rootstocks on growth and productivity under glasshouse conditions. Asian Journal of Agricultural Research 3(2): 47-54.

[28] Morales-Garcia,D., Stewart,K.A., Seguin,P., Madramootoo, C. (2010): Saline drip irrigation and polyethylene mulch on yield and water use efficiency of Bell Peppers. International Journal of Vegetable Science 16: 3-14.

[29] Munns, R., Tester, M. (2008): Mechanisms of salinity tolerance. Annu. Rev. - Plant Biol. 59: 651-681.

[30] Öztekin, G.B. (2009): Aş11 domates bitkilerinde tuz stresine karş1 anaçların etkisi (Response of tomato rootstocks to salinity stress). - [Ph.D. Thesis.] Ege University in Turkey, p.: 1-342.

[31] Öztekin, G.B., Tuzel, Y., Tuzel, I.H. (2013): Does mycorrhiza improve salinity tolerance in grafted plants. - Scientia Horticulturae 149: 55-60.

[32] Qaryouti, M.M., Qawasmi W., Hamdan H., Edwan M. (2007): Tomato fruit yield and quality as affected by grafting and growing system. - Acta Horticulturae 741: 199-206.

[33] Ranatunga, C.L., Jayaweera, H.H.E., Ariyaratne, T.R. (2008): Comparison of firmness of tomato varieties grown in Sri Lanka. - Proceedings of the Technical Sessions 24: 1-5.

[34] Rouphael, Y., Schwarz, D., Krumbein, A., Colla, G. (2010): Impact of grafting on product quality of fruit vegetables. - Scientia Horticulturae 127: 172-179.

[35] Sanchez-Rodriguez, E., Leyva, R., Constan-Aguilar, C., Romero, L., Ruiz, J.M. (2012): Grafting under water stress in tomato cherry: improving the fruit yield and quality. Annals of Applied Biology 161: 302-312. 
[36] SAS (1995): SAS/STAT User's Guide. Version 6.12. - SAS Institute, Cary, North Carolina.

[37] Savvas, D., Colla, G., Rouphael, Y., Schwarz, D. (2010): Amelioration of heavy metal and nutrient stress in fruit vegetables by grafting. - Scientia Horticulturae 127: 156-161.

[38] Savvas, D., Savva, A., Ntatsi, G., Ropokis, A., Karapanos, I., Krumbein, A., Olympios, C. (2011): Effects of three commercial rootstock on mineral nutrition, fruit yield and quality of salinized tomato. - J. Plant Nutr. Soil Sci. 174: 154-162.

[39] Semiz, G.D., Suarez, D.L. (2015):Tomato salt tolerance: Impact of grafting and water composition an yield and ion relations. - Turkish Journal of Agriculture and Forestry 39:876-886.

[40] Sonneveld, C., Welles, G.W.H. (1988): Yield and quality of rockwool-grown tomatoes as affected by variations in EC-value and climatic conditions. - Plant and Soil 111: 37-42.

[41] Turhan, A., Ozmen, N., Serbeci, M.S., Seniz, V. (2011): Effects of grafting on different rootstocks on tomato fruit yield and quality. - Hort. Sci. 38(4): 142-149.

[42] Tüzel, İ.H., Tüzel, Y., Gül, A., Eltez, R.Z. (2001): Effects of EC level of the nutrient solution on yield and fruit quality of tomatoes. - Acta Horticulturae 559(II): 587-592.

[43] Voutsela, S., Yarsi, G., Petropoulos, S.A., Khan, E.M. (2012): The effect of grafting of five different rootstocks on plant growth and yield of tomato plants cultivated outdoors and indoors under salinity stres. - African Journal of Agricultural Research 7(41): 55535557.

[44] Vrcek, V., Samobor, V., Bojic, M., Medic-Saric, M., Vukobratovic, M. (2011): The effect of grafting on the antioxidant properties of tomato (Salanum lycopersicum L.). - Spanish Journal of Agricultural Research, 9(3): 844-851.

[45] Wahb-Allah, M.A. (2014); Effectiveness of grafting fort he improvement of salinity and drought tolerance in tomato (Solanum lycopersicon L.) - Asian Journal of Crop Science 6(2): 112-122.

[46] Wu, M., Buck, J.S., Kubota, C. (2004): Effects of nutrient solution EC, plant microclimate and cultivars on fruit quality and yield of hydroponic tomatoes (Lycopersicon esculentum). - Acta Horticulturae, 659: 541-547.

[47] Zhang, P., Senge, M., Dai, Y. (2016): Effects of salinity stress on growth, yield, fruit quality and water use efficiency of tomato under hydroponics system. - Reviews in Agricultural Science 4: 46-55. 